\title{
Benchmark values for the net proton number fluctuations
}

\author{
Boris Tomášik* \\ Fakulta prírodných vied, Univerzita Mateja Bela, Banská Bystrica, Slovakia \\ and \\ Fakulta jaderná a fyzikálně inženýrská, České vysoké učení technické v Praze, Prague, Czechia \\ E-mail: boris.tomasikecern.ch
}

\section{Ivan Melo}

Fakulta elektrotechniky a informačných technológií, Žilinská univerzita, Žilina, Slovakia

\section{Lukáš Lafférs}

Fakulta prírodných vied, Univerzita Mateja Bela, Banská Bystrica, Slovakia

\section{Marcus Bleicher}

Frankfurt Institute for Advanced Studies, Johann Wolfgang Goethe-Universität,

Frankfurt am Main, Germany,

and

Institut für Theoretische Physik, Johann Wolfgang Goethe-Universität,

Frankfurt am Main, Germany

\begin{abstract}
We formulate a baseline model for the net-proton number fluctuations in ultrarelativistic heavyion collisions. Our model includes total baryon number conservation, fluctuations of the participating nucleon number, limited acceptance, and a possibility that isospin is remembered by the participating wounded nucleons. Most importantly, we formulated the model as consisting of two components: wounded nucleons and the produced nucleon-antinucleon pairs. Those two components have different rapidity distributions. Owing to this feature we obtain also predictions for the rapidity dependence of the cumulants of the net-proton number distribution and their ratios.
\end{abstract}

Corfu Summer Institute 2018 "School and Workshops on Elementary Particle Physics and Gravity" (CORFU2018)

31 August - 28 September, 2018

Corfu, Greece

${ }^{*}$ Speaker. 


\section{Introduction}

Statistical physics arguments lead to the conclusions about strongly interacting matter, that in the vicinity of the critical point of the phase diagram the susceptibilities of higher orders should strongly depend on temperature and the baryon chemical potential $\mu_{B}[1,2,3,4,5,6]$. The susceptibilities with respect to $\mu_{B}$ represent themselves in the cumulants of the net-baryon number distribution $[2,6]$. In principle, they can be even calculated from the first principles by simulating the QCD partition function on the lattice [7, 8]. These arguments and calculations are intrinsically connected with the grand-canonical ensemble of systems.

Unfortunately, the baryon number fluctuations, as they are elegantly treated in the grandcanonical formalism, cannot be directly measured in an experiment. Three major reasons make this impossible:

- In real collisions, baryon number is always conserved. Hence, if the whole phase-space would be covered by detectors that would register all baryons and antibaryons with perfect efficiency, there would be no fluctuations. On the other hand, if the detectors cover only a part of the phase-space, then fluctuations may arise just from the binomial distribution of the appearance of a particle in the acceptance window $[9,10]$.

- It is experimentally impossible to investigate a set of heavy-ion collisions with always exactly the same number of nucleons participating in the collisions. This brings in another source of baryon number fluctuations. It has to be taken into account also in theoretical simulations which are compared to experimental data. Another possibility is to filter out such fluctuations from the experimental data my means of unfolding $[11,12]$.

- Not all baryons (and antibaryons) can be detected. A typical detector does not register neutrons. Therefore, the net-proton number is usually measured as a good proxy for the net baryon number. The argument in favour of this proxy is usually based on the high rate of the isospin-changing reactions that can change protons into neutrons and vice versa $[13,14]$. Note, however, that such arguments may be of limited validity at the lowest collision energies within the RHIC Beam Energy Scan programme.

The less-than-perfect acceptance of the detectors also acts as a source of fluctuations. This problem has been addressed in the literature and we will not analyse it here [15, 16, 17, 18]. Instead, we assume that the detector efficiency is $100 \%$.

In this study we investigate the influence of the effects mentioned above on the net-baryon and the net-proton number fluctuations. Two new points are introduced in our treatment.

- We simulate the participating baryons and the produced $B \bar{B}$ pairs with rapidity distributions that depend on the collision energy. This, combined with the rapidity window fixed for all collision energies (since it is really fixed by the actual detector), leads to the collision energy dependence of the fluctuations. The rapidity distributions are constructed with an eye on the experimental data $[23,24]$. The distribution of the wounded nucleons is different from the distribution of the produced $B \bar{B}$ pairs. 
- Since for lower collision energies the isospin randomisation may become ineffective, we also explore the possibility that the wounded nucleons remember their isospin.

We study the fluctuations as functions of the size of the acceptance window in rapidity but also as a function of the position of the acceptance window. The latter is relevant as a baseline study for the recent proposal to look for the position of the critical point by measuring fluctuations at slightly forward rapidities, where the net baryon density may be higher than at midrapidity [19]. This is doable for us owing to the implemented rapidity distributions of the wounded nucleons.

\section{The model}

Our results will be based on Monte Carlo simulations that will assign the baryons and antibaryons their rapidity. First of all, we respect the baryon number conservation. Only protons, neutrons, and their antiparticles are included into our simulations. From the colliding gold nuclei only a part of the incoming nucleons participate in the collision.

The number of wounded nucleons is determined with the help of GLISSANDO [20, 21, 22]. We first create a sample of minimum bias collisions for each of the investigated collision energies. Then we select centrality classes based on the relative deposited strength (RDS) which should be proportional to the produced multiplicity

$$
M \propto \frac{1-\alpha}{2} N_{w}+\alpha N_{b i n},
$$

where the parameter $\alpha$ increases with the collision energy. It has been set by fitting the centrality dependence of the multiplicity in Au+Au collisions at $\sqrt{s_{N N}}=19.6 \mathrm{GeV}$ and $200 \mathrm{GeV}$ and interpolating for other energies with the logarithmic dependence

$$
\alpha\left(\sqrt{s_{N N}}\right)=\alpha_{0}+\alpha_{1} \ln \sqrt{s_{N N}} .
$$

This method is closest to what is done in real experiment.

One of the features of our model, which can be turned on and off, is the isospin memory of wounded nucleons. This may be (partially) present in collisions at the lowest energies. The isospin of a wounded nucleon is set in such a way that one never can obtain more protons (or neutrons) than there are in the incoming nucleus. This means that if $N_{p}$ wounded protons and $N_{n}$ wounded neutrons have been generated, then the probability that the next wounded nucleon will be a proton is $\left(Z-N_{p}\right) /\left(A-N_{p}-N_{n}\right)$, where $A$ and $Z$ refer to gold nucleus. The number of wounded protons is then distributed according to hypergeometric distribution.

In addition to the wounded nucleons there are also produced pairs of nucleons and antinucleons. The number of pairs fluctuates according to Poisson distribution, with its mean proportional to the number of wounded nucleons

$$
\mu_{B \bar{B}}=\frac{d N_{\bar{p}}}{d y} y_{m} \frac{N_{w}}{\left\langle N_{w}\right\rangle},
$$

where $d N_{\bar{p}} / d y$ is the rapidity density of antiprotons measured at given energy and centrality [23, 24], $y_{m}$ is the width of the whole rapidity distribution of the produced $B \bar{B}$ pairs, $N_{w}$ is the number of wounded nucleons in the particular event, and $\left\langle N_{w}\right\rangle$ is the mean number of wounded nucleons at 
given collision energy and centrality, as determined by GLISSANDO. The isospin is assigned with equal probabilities for (anti)protons and (anti)neutrons. Antinucleons are, of course, only present among the produced $B \bar{B}$ pairs.

Not the whole rapidity region is covered by the detectors, thus only a part of the (anti)nucleons with rapidities in the experimentally accepted window is assumed to be registered.

It is a crucial feature of this model, that the relative abundance of wounded and produced nucleons depends on the collision energy, centrality, and the acceptance window in rapidity.

Finally, let us specify the rapidity distributions. Wounded nucleons partially remember their original rapidity, so we parametrize their rapidity distribution with a double Gaussian function

$$
\frac{d N_{w}}{d y}=\frac{N_{w}}{2 \sqrt{2 \pi \sigma_{y}^{2}}}\left\{\exp \left(-\frac{\left(y+y_{m}\right)^{2}}{2 \sigma_{y}^{2}}\right)+\exp \left(-\frac{\left(y-y_{m}\right)^{2}}{2 \sigma_{y}^{2}}\right)\right\} .
$$

We set the width $\sigma_{y}=0.8$, and $N_{w}$ is the total number of wounded nucleons in the given event. The shifts $y_{m}$ are determined from data. We use the fact that wounded protons make up the difference between observed protons and antiprotons. Thus the number of the observed net protons in a given rapidity window $\left(-y_{b}, y_{b}\right)$ can be determined

$$
N_{p-\bar{p}}=\frac{Z}{A} \int_{-y_{b}}^{y_{b}} \frac{d N_{w}}{d y} d y .
$$

We use data for $y_{b}=0.25$ measured at different collision energies by the STAR collaboration $[23,24]$.

For the produced nucleon-antinucleon pairs we assume that they are produced mainly around midrapidity. To soften sharp edges of their distribution we use the Woods-Saxon distribution

$$
\frac{d N_{B \bar{B}}}{d y}=N_{B \bar{B}} \frac{C}{1+\exp \left(\frac{|y|-y_{m}}{a}\right)},
$$

where $C$ is the proper normalisation constant $C=\left(2 a \ln \left(e^{y_{m} / a}+1\right)\right)^{-1}$, and we choose $a=\sigma_{y} / 10$. The number of pairs $N_{B \bar{B}}$ is determined from data: note that this is the only source of antiprotons in our model. Thus we set the parameters so that we can reproduce the measured number of antiprotons $N_{\bar{p}}$ in the interval $|y|<y_{b}=0.25[23,24]$

$$
N_{\bar{p}}=\frac{1}{2} \int_{-y_{b}}^{y_{b}} \frac{d N_{B \bar{B}}}{d y} d y
$$

where the factor $1 / 2$ in front of the integral stands for taking only antiprotons and no antineutrons.

The pairs of nucleons and antinucleons are generated so that their mutual distances in rapidity are distributed exponentially with the correlation length 1 . The correlation length may have influence on the fluctuations [25] and we plan to investigate this effect in the future.

With this model we determine the central moments $\mu_{i}$ up to the fourth order and the volumeindependent ratios of cumulants $\chi_{i}$

$$
\begin{aligned}
S \sigma & =\frac{\chi_{3}}{\chi_{2}}=\frac{\mu_{3}}{\mu_{2}} \\
\kappa \sigma & =\frac{\chi_{4}}{\chi_{2}}=\frac{\mu_{4}}{\mu_{2}}-3 \mu_{2} \\
\frac{\kappa \sigma^{4}}{\bar{n}} & =\frac{\chi_{4}}{\chi_{1}}=\frac{\mu_{4}-3 \mu_{2}^{2}}{\mu_{1}},
\end{aligned}
$$



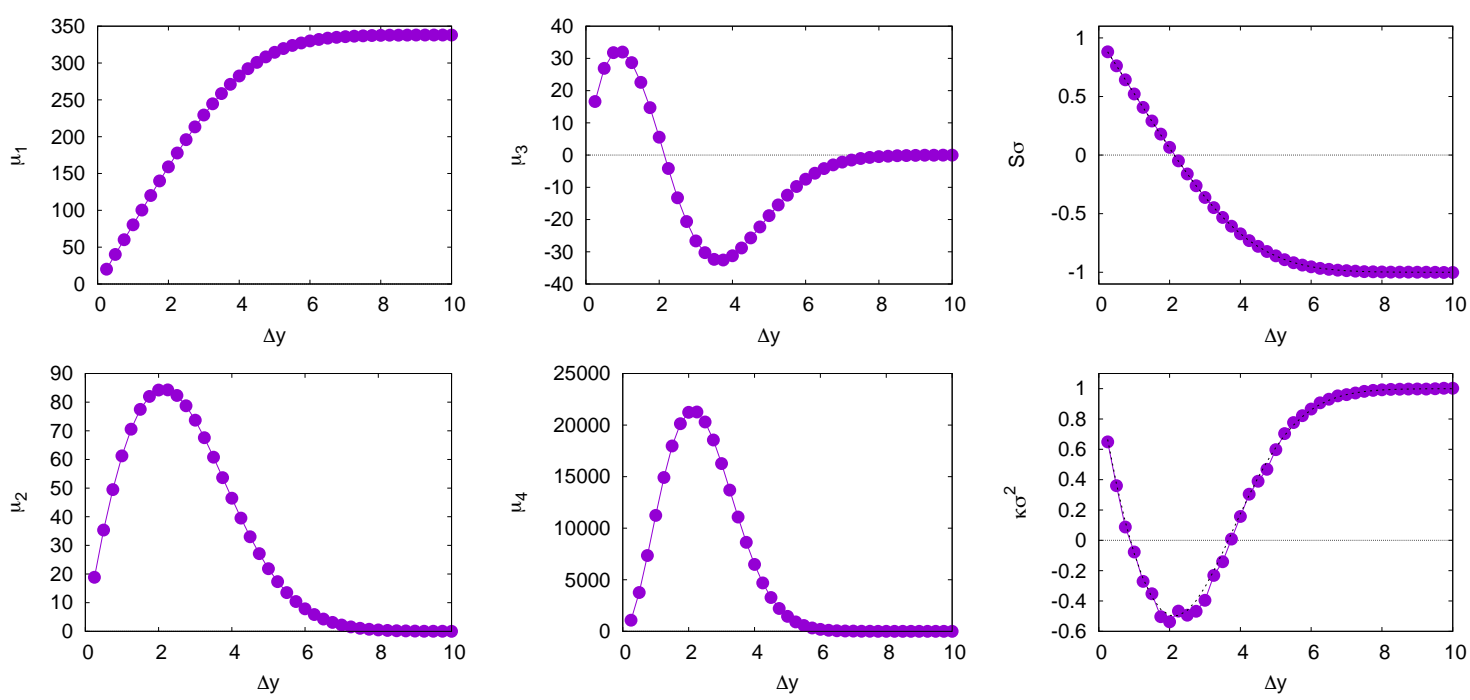

Figure 1: Central moments of the baryon number distribution and the volume-independent ratios $S \sigma$ and $\kappa \sigma^{2}$ as functions of the rapidity window $\Delta y$. Parameter settings: $N_{w}=338, y_{m}=1.019$, and $N_{B \bar{B}}=16.946$. The dotted curves in the two right panels show the dependences (3.1) and (3.2).

where we have used $\sigma$ for the width, $S$ for the skewness, and $\kappa$ for the kurtosis of the multiplicity distribution.

\section{A warm-up exercise: baryon number conservation and modifications}

Before we embark on more realistic simulation, let us test the model and present the mere effect of baryon number conservation and limited acceptance. We inspect a sample of $5.10^{7}$ events with fixed number of wounded nucleons $N_{w}=338$ and other parameters set to reproduce $\mathrm{Au}+\mathrm{Au}$ collisions at $\sqrt{s_{N N}}=19.6 \mathrm{GeV}: y_{m}=1.019$ and $N_{B \bar{B}}=16.946$.

In Figure 1 we plot the central moments and volume independent cumulant ratios of the net baryon number distribution, as functions of the width of the acceptance window $\Delta y$ around midrapidity. We show the central moments since they are the primary quantities which could be determined experimentally and the other ratios are based on them. Thus one can relate the behaviour of the $\Delta y$ dependences of the scaled skewness and the scaled kurtosis to the behaviour of the central moments. As $\Delta y$ increases, the mean value $\mu_{1}$ saturates at $N_{w}$ while the higher moments go to 0 as there is no room for fluctuations due to baryon number conservation. The dependences are actually described by the binomial distribution where the probability $p$ equals $\mu_{1}(\Delta y) / N_{w}$. This is demonstrated in the plots for the scaled skewness $S \sigma$ and the scaled kurtosis $\kappa \sigma^{2}$. The simulated points are compared to the curves obtained for the binomial distribution

$$
\begin{aligned}
S \sigma & =1-2 p=1-2 \frac{\mu_{1}(\Delta y)}{N_{w}} \\
\kappa \sigma^{2} & =1-6 p+6 p^{2}=1-6 \frac{\mu_{1}(\Delta y)}{N_{w}}+6\left(\frac{\mu_{1}(\Delta y)}{N_{w}}\right)^{2} .
\end{aligned}
$$

The observed dependences result just from the rapidity distribution of baryons and antibaryons combined with the binomial distribution of the number of accepted nucleons. 

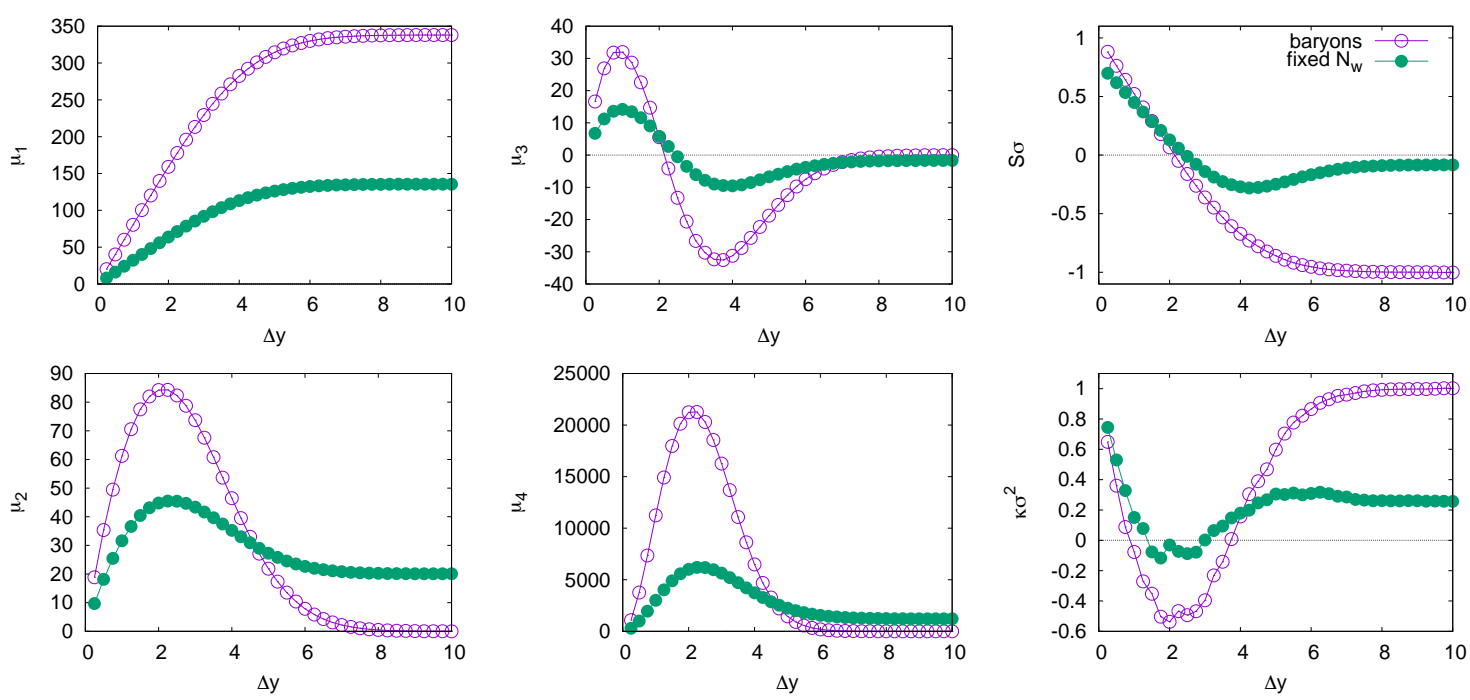

Figure 2: Central moments of the net proton number distribution and the volume-independent ratios $S \sigma$ and $\kappa \sigma^{2}$ as functions of the rapidity window $\Delta y$. Parameter settings as in Fig. 1. Curves for the net proton number (full green circles) are compared with the curves for net baryons (open purple circles) shown in Fig. 1. The number of wounded nucleons is fixed in these simulations.

The third central moment and consequently the skewness is positive for small $\Delta y$ and then turns negative. This means, that in small rapidity windows numbers larger than the mean are rather populated. As the rapidity window gets larger, the total baryon number limits the possible observed numbers and they are more often populated below the mean.

Let us next look at how the results are modified, if now instead of baryons one measures the fluctuations of net protons. They are shown in Fig. 2. The rough features of the baryon number distribution are inherited here. However, just by looking at the net protons-whose number is not conserved - there are qualitative differences from the net baryons and the binomial distribution is no longer applicable. This is due to two reasons: firstly, the produced nucleon-antinucleon pairs populate protons and neutrons with equal probability. Secondly, also the wounded nucleons may change isospin. The result is that now the total number of net protons is not fixed and fluctuates from event to event. This is seen in the behaviour of the higher central moments which saturate at a non-vanishing values for large rapidity windows. The asymptotic values of $S \sigma$ and $\kappa \sigma^{2}$ are modified, as well.

The next complication comes from the fluctuation of the number of participating nucleons. Centrality classes in an experiment are usually determined from the produced multiplicity. That usually scales with the relative deposited strength as defined in eq. (2.1) which can be evaluated in GLISSANDO. Within the 0-5\% percentile with highest RDS among all events, which corresponds to the experimental class of $0-5 \%$ centrality, the number of wounded nucleons fluctuates. Figure 3 shows how this influences the net-proton number fluctuations. In general, all central moments characterising fluctuations grow considerably with respect to the simulation with fixed $N_{w}$. Nevertheless, some qualitative features of their dependence on $\Delta y$ remain, e.g., the third moment flips the sign at the same $\Delta y$ as it did for fixed $N_{w}$. These features are then reflected also in $S \sigma$ and $\kappa \sigma^{2}$. 

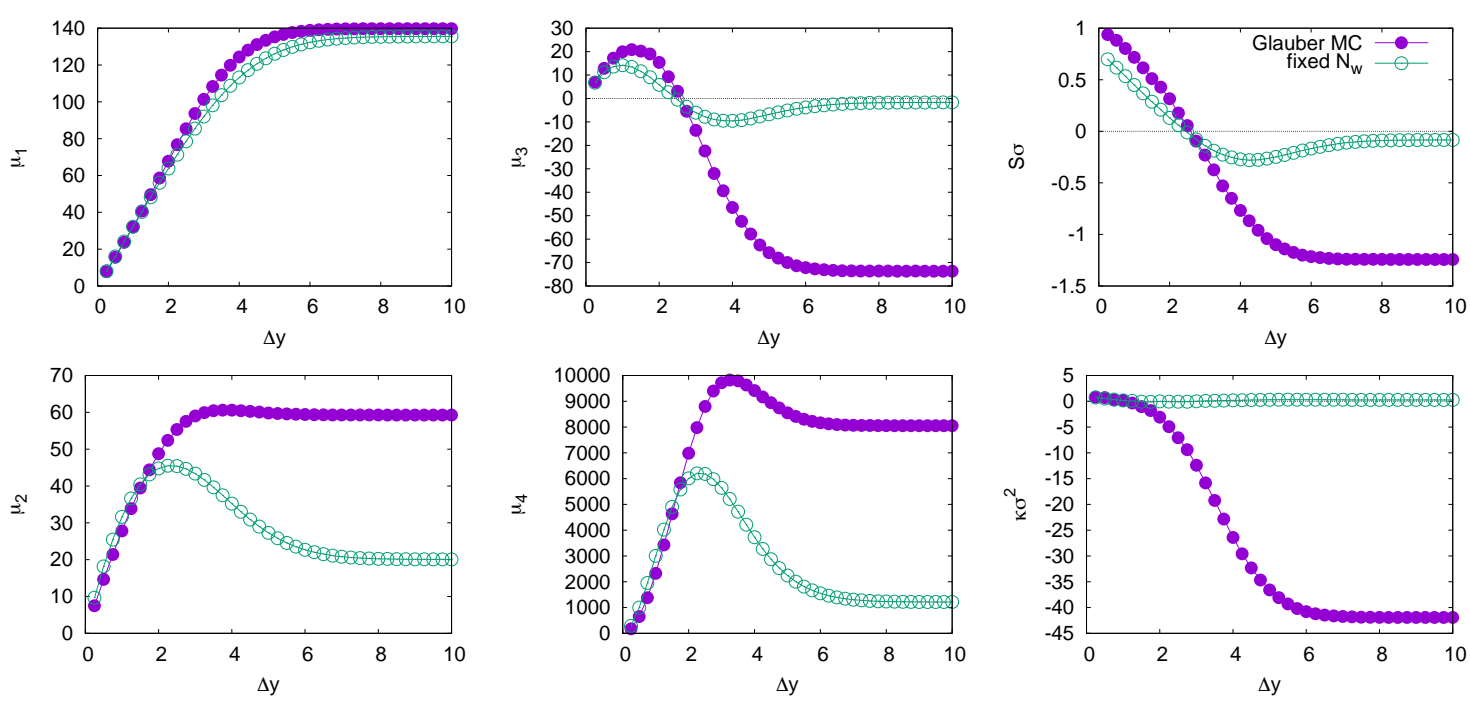

Figure 3: Central moments of the net proton number distribution and the volume-independent ratios $S \sigma$ and $\kappa \sigma^{2}$ as functions of the rapidity window $\Delta y$. Parameter settings as in Figs. 1 and 2. Open green symbols show the dependences for $N_{w}$ fixed to 338 (same curves as in Fig. 2) and full purple symbols show the dependences for fluctuating $N_{w}$ within the $0-5 \%$ centrality class as determined from $1.2 \times 10^{6}$ events generated by GLISSANDO.

\section{Results}

Figures 1-3 demonstrate the influence of the three basic effects on the observed fluctuations: baryon number conservation (Fig. 1), measurement of only protons (Fig. 2), and fluctuations of the interacting volume (Fig. 3). From Fig. 3 one would conclude that the latter influence is tremendous. However, no real detector in the current experiments covers 10 units of rapidity. Therefore, in Figure 4 we zoom into a narrower $\Delta y$-interval of 2 units, which may realistically be covered by the central detector, e.g. in the STAR experiment at RHIC. Here, the only large difference among the central moments shows up for the third moment: results from Glauber MC simulations lead to larger $\mu_{3}$. This is then translated in about a constant increase of $S \sigma$ by 0.25 in the whole interval of $\Delta y$. Up to $\Delta y=1$, the values of $\kappa \sigma^{2}$ for fluctuating and for fixed $N_{w}$ are practically identical. However, they start to depart from each other strongly beyond this limit.

An interesting suggestion [19] has been presented also at this conference [26] that the critical point might be sought by inspecting the net-proton number fluctuations at different rapidities as an alternative to different collision energies. Our model includes the feature, that rapidity distributions of protons and antiprotons are different. Hence, we can make predictions for the rapidity dependence of the net-proton number fluctuations in absence of any critical behaviour. This is shown in Figure 5. Simulations were done for a set of parameters that correspond to $\sqrt{s_{N N}}=19.6 \mathrm{GeV}$. Results have been extracted for a rapidity window of $\Delta y=0.5$, which is put in different positions along the rapidity distribution. Figure 5 shows the dependences on the central value of the rapidity window. We compare the results obtained for fixed $N_{w}=338\left(2 \times 10^{7}\right.$ events in the sample) with events from $0-5 \%$ central collisions according to GLISSANDO Glauber MC $\left(1.2 \times 10^{6}\right.$ events). The central moments calculated for fixed $N_{w}$ generally decrease as we go towards higher $y$. In con- 

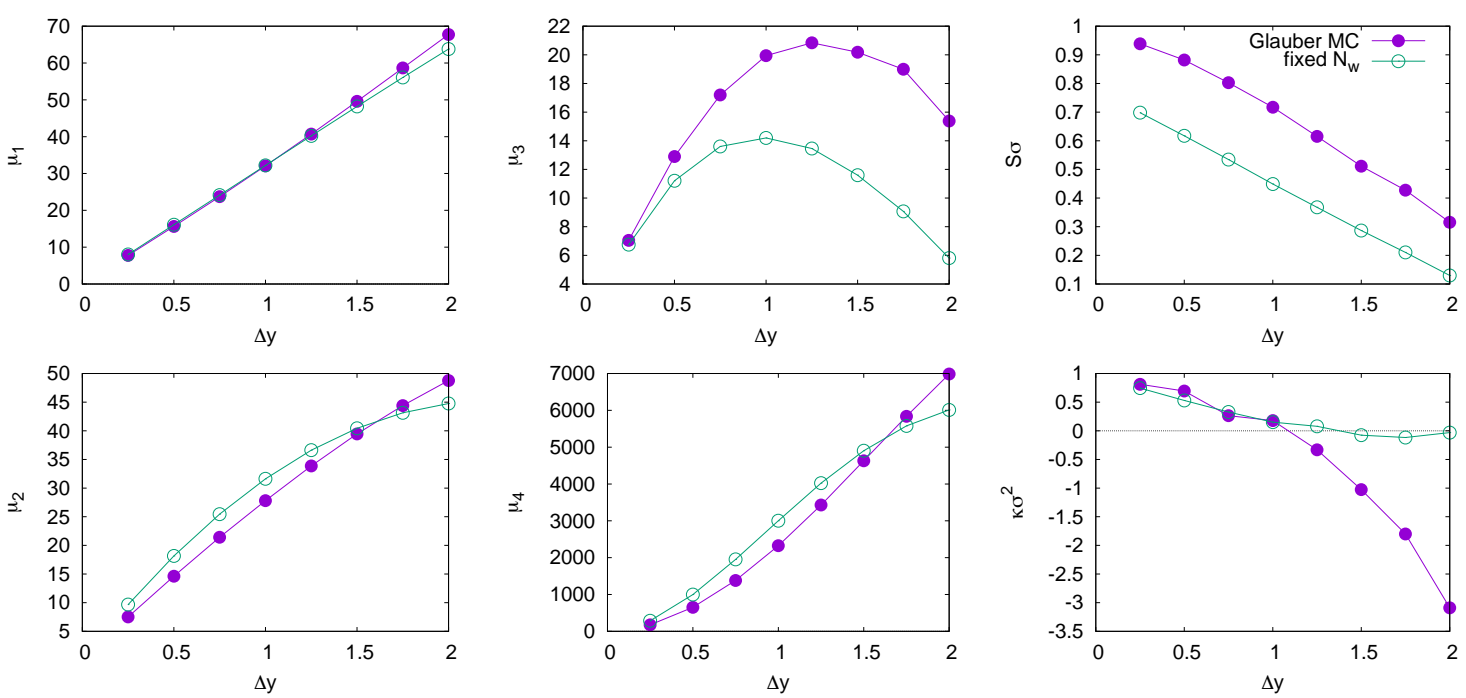

Figure 4: Same as Figure 3 but zoomed into smaller interval of $\Delta y$.
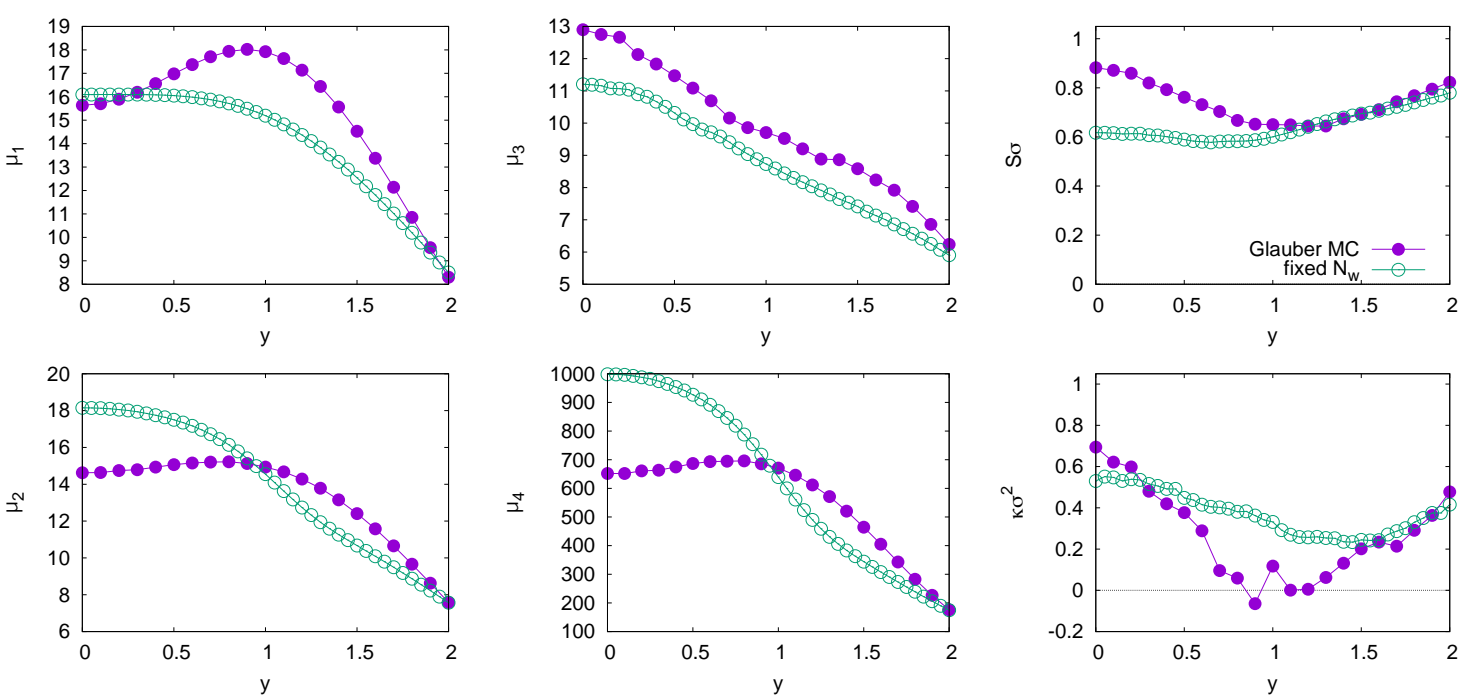

Figure 5: Rapidity dependence of the central moments and volume-independent ratios $S \sigma$ and $\kappa \sigma^{2}$ of the net-proton distribution. Parameter settings: $N_{w}=338, y_{m}=1.019$, and $N_{B \bar{B}}=16.946$ (like in Fig. 1). Open green symbols show results for fixed $N_{w}=338$, full purple circles show results from $1.2 \times 10^{6}$ of $0-5 \%$ central Glauber MC events generated by GLISSANDO.

trast to that, the curves for $\mu_{1}, \mu_{2}$, and $\mu_{4}$ simulated with Glauber MC show peaks around $y=y_{m}$, i.e., where the antiprotons die out. Note that the antiproton rapidity distribution is cut more sharply than that of wounded protons. This results in a dip in both $S \sigma$ and $\kappa \sigma^{2}$ as functions of $y$ around $y=1$.

Since $y_{m}$ increases with the collision energy, we would also expect that the position of the dip would move to higher values of $y_{m}$. This is confirmed in Figure 6, where we show $S \sigma, \kappa \sigma^{2}$, and $\chi_{4} / \chi_{1}$ as functions of $y$ for three collision energies. The parameters used in the simulations for all energies are summarised in Table 1. In the Figure we only show results from Glauber MC 

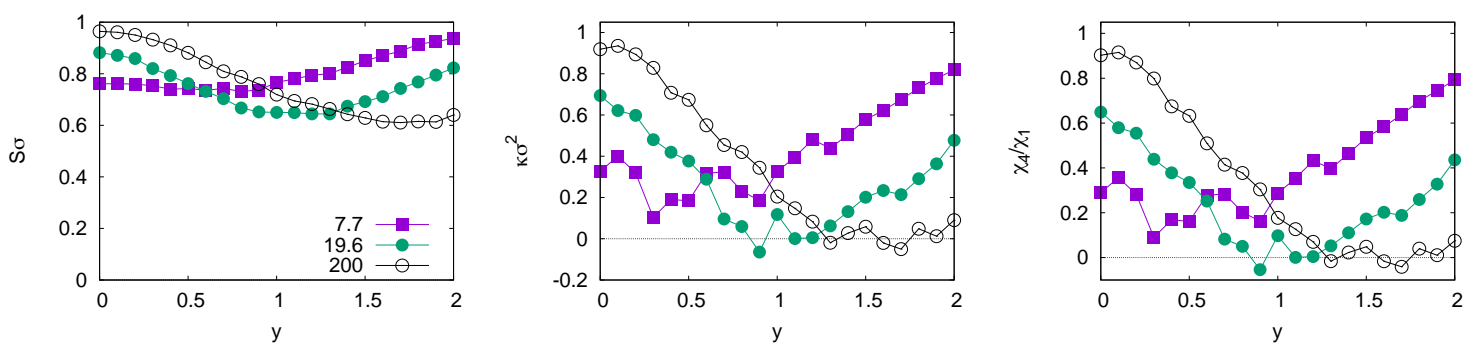

Figure 6: Rapidity dependence of central moments, $S \sigma$, and $\kappa \sigma^{2}$ for three different collision energies, as indicated in the legend. For each energy $1.2 \times 10^{6}$ events in $0-5 \%$ centrality class generated by GLISSANDO have been analysed.

version of our model. Unfortunately, the statistics of $1.2 \times 10^{6}$ events is not sufficient to avoid large statistical errors in the curves for the fourth order. Nevertheless, one can see that this simple baseline model predicts rather strong dependence on $y$ of the fourth order cumulant ratios which for $\sqrt{s_{N N}}=200 \mathrm{GeV}$ start around 1 and fall down all the way to 0 at $y=1.2$. The decrease is less severe when the collision energy decreases.

In order to present the centrality dependence of the fluctuations we turn back to $\sqrt{s_{N N}}=$ 19.6 GeV. Selected results are shown in Fig. 7: $S \sigma$ and $\kappa \sigma^{2}$ as functions of the number of wounded nucleons $N_{w}$. The number $N_{w}$ is either fixed or we use for plotting the mean number from GLISSANDO for the selected centrality class. With these results we also show the influence of wounded nucleon isospin randomisation or isospin memory. We investigated the influence of this effect on the simulations with fixed $N_{w}$. The centrality dependence appears flat with randomised isospins. The effect of isospin memory is most pronounced in most central collisions since it is here that the limit on incoming proton number kicks in most strongly. As a result, the third and the fourth cumulant are slightly lowered in central collisions. The isospin memory has also been assumed in the simulations with the Glauber MC model. Although the statistics of $5 \times 10^{5}$ events for each centrality still leads to large statistical uncertainties, we see that these fluctuations increase $S \sigma$ by about 0.1 and $\kappa \sigma^{2}$ by about 0.2 .

Finally, we investigated the dependence of the moments on the collision energy in Figure 8. (Note that model parameters for all energies have been summarised in Table 1.) We show results

Table 1: Parameters of the model for different collision energies.

\begin{tabular}{|c|c|c|}
\hline$\sqrt{s_{N N}}[\mathrm{GeV}]$ & $y_{m}$ & $N_{B \bar{B}}$ \\
\hline 7.7 & 0.519 & 0.8265 \\
11.5 & 0.770 & 4.4790 \\
19.6 & 1.019 & 16.946 \\
27 & 1.128 & 27.1070 \\
39 & 1.308 & 44.4262 \\
62.4 & 1.384 & 75.2842 \\
200 & 1.665 & 177.794 \\
\hline
\end{tabular}



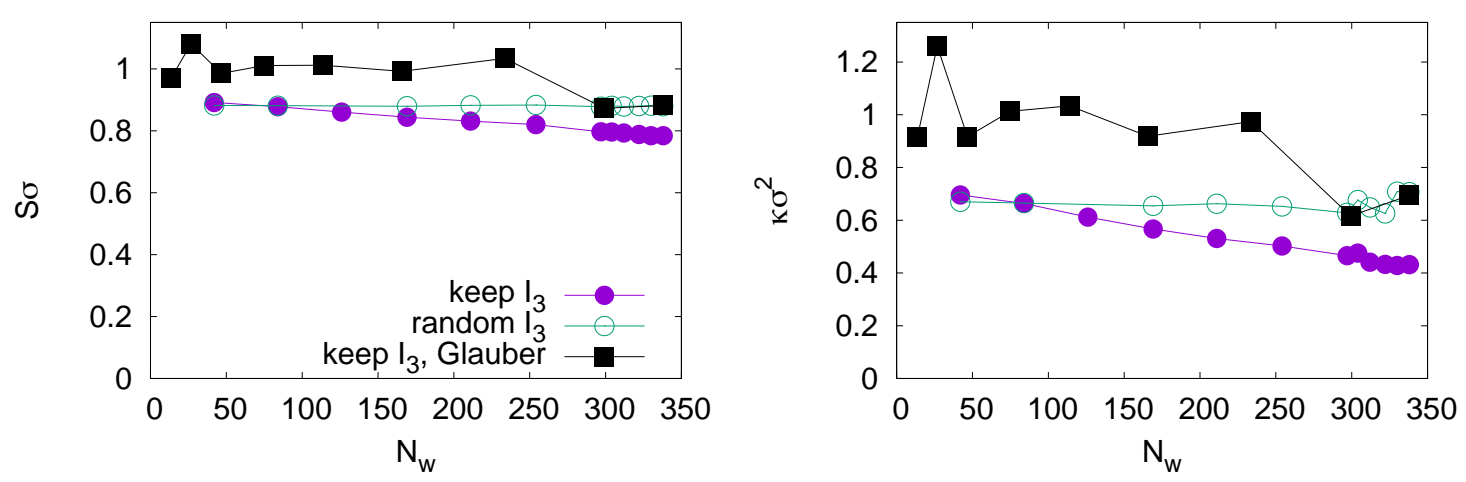

Figure 7: Centrality dependence of $S \sigma$ and $\kappa \sigma^{2}$ for parameters corresponding to central $\mathrm{Au}+\mathrm{Au}$ collisions at $\sqrt{s_{N N}}=19.6 \mathrm{GeV}$. Full purple circles: fixed $N_{w}$ at values corresponding to mean at given centrality class and wounded nucleons remember their original isospin. Open green circles: fixed $N_{w}$ for given centrality class and wounded nucleons have their isospins randomised. Solid black squares: Glauber MC fluctuating $N_{w}$ corresponding to $0-5 \%$ most central with wounded nucleons remembering their original isospin.
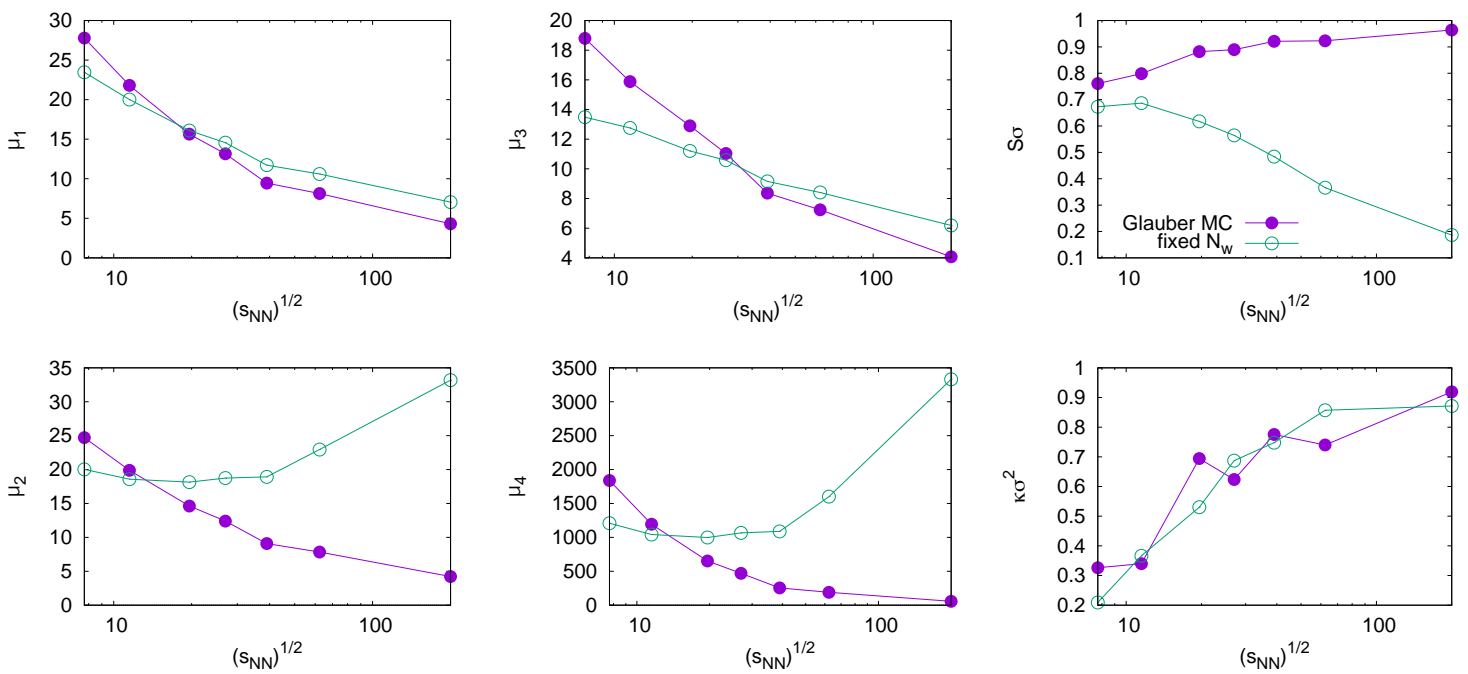

Figure 8: Dependence of the central moments of the net=proton number distribution, $S \sigma, \kappa \sigma^{2}$ on $\sqrt{s_{N N}}$ in $\mathrm{Au}+\mathrm{Au}$ collisions. Open green circles: simulations with fixed $N_{w}$. Full purple circles: simulations with $0-5 \%$ most central collisions based on GLISSANDO Glauber MC.

for $0-5 \%$ central collisions. Since we are showing results for the central rapidity window of $\Delta y=$ 0.5 , the baryon-antibaryon asymmetry decreases as the collision energy goes up. The relative contribution of produced $B \bar{B}$ pairs is increasing. This is demonstrated by $\sqrt{s_{N N}}$ dependence of $\mu_{1}$. In general, all higher central moments decrease, as well, if we look at the results obtained for fluctuating $N_{w}$. For $\mu_{2}$ and $\mu_{4}$ we see that the curves for fixed $N_{w}$ (i.e. which do not include all baseline effects) increase together with $\sqrt{s_{N N}}$. This behaviour is reflected in $S \sigma$ which starts below 0.8 at low collision energies and approaches 1 as $\sqrt{s_{N N}} \rightarrow 200 \mathrm{GeV}$ for Glauber MC simulations, but is strongly decreased in fixed $N_{w}$ results. The scaled kurtosis $\kappa \sigma^{2}$, on the other hand, does not change when $N_{w}$ fluctuations are turned on and off, since $\mu_{2}$ and $\mu_{4}$ behave similarly, here. 
The scaled kurtosis starts at very low values for $\sqrt{s_{N N}}=7.7 \mathrm{GeV}$ and grows together with $\sqrt{s_{N N}}$. Unfortunately, this is exactly opposite to the observed experimental result [27] which shows a huge increase of $\kappa \sigma^{2}$ as $\sqrt{s_{N N}} \rightarrow 7.7 \mathrm{GeV}$. Hence, our result just highlights the uncommonness of the measured value.

\section{Conclusions}

We have set up a benchmark model which includes many non-critical effects that influence the fluctuations of net-proton number. Generally, it is always necessary to look at such trivial predictions when interpreting the measured data.

In particular, our model allowed to look at the dependence of the moments of the net-proton number distribution on the rapidity of the centre of the acceptance window. Our predictions, presented in Figures 5 and 6, are thus complementary to the proposal to use the rapidity dependence for looking for the critical point of the phase diagram [19].

We have also looked at the effect of the wounded nucleons remembering their original isospin. Such a mechanism may be present in nuclear collisions at $\sqrt{s_{N N}}$ of the order of a few $\mathrm{GeV}$, because there the isospin randomisation may become ineffective.

Nevertheless, the presented baseline effects are not sufficient to explain all observed features of the data; in particular the huge enhancement of $\kappa \sigma^{2}$ towards $\sqrt{s_{N N}}=7.7 \mathrm{GeV}$ is not reproduced by this model. One has to, however, always keep them in mind when interpreting any measured result.

\section{Acknowledgements}

This work was supported by the grant 17-04505S of the Czech Science Foundation (GAČR) and the grant VEGA 1/0348/18 (Slovakia). International collaboration was supported by collaboration grant in framework of the German-Slovak PPP programme and the COST Action CA15213 THOR.

\section{References}

[1] Y. Hatta and M. A. Stephanov, Phys. Rev. Lett. 91 (2003) 102003 Erratum: [Phys. Rev. Lett. 91 (2003) 129901]

[2] C. Athanasiou, K. Rajagopal and M. Stephanov, Phys. Rev. D 82 (2010) 074008

[3] R. V. Gavai and S. Gupta, Phys. Lett. B 696 (2011) 459.

[4] M. A. Stephanov, Phys. Rev. Lett. 107 (2011) 052301.

[5] S. Gupta, X. Luo, B. Mohanty, H. G. Ritter and N. Xu, Science 332 (2011) 1525

[6] M. A. Stephanov, Phys. Rev. Lett. 102 (2009) 032301.

[7] A. Bazavov et al. [HotQCD Collaboration], Phys. Rev. D 96 (2017) no.7, 074510.

[8] S. Borsányi, Z. Fodor, J. N. Guenther, S. K. Katz, K. K. Szabo, A. Pasztor, I. Portillo and C. Ratti, JHEP 1810 (2018) 205

[9] P. Braun-Munzinger, A. Rustamov and J. Stachel, Nucl. Phys. A 960 (2017) 114 
[10] A. Bzdak and V. Koch, Phys. Rev. C 96 (2017) no.5, 054905.

[11] X. Luo, J. Xu, B. Mohanty and N. Xu, J. Phys. G 40 (2013) 105104.

[12] T. Sugiura, T. Nonaka and S. Esumi, arXiv:1903.02314 [nucl-th].

[13] M. Kitazawa and M. Asakawa, Phys. Rev. C 85 (2012) 021901.

[14] M. Kitazawa and M. Asakawa, Phys. Rev. C 86 (2012) 024904, Erratum: [Phys. Rev. C 86 (2012) 069902].

[15] A. Bzdak and V. Koch, Phys. Rev. C 91 (2015) no.2, 027901 doi:10.1103/PhysRevC.91.027901 [arXiv:1312.4574 [nucl-th]].

[16] X. Luo, Phys. Rev. C 91 (2015) no.3, 034907 Erratum: [Phys. Rev. C 94 (2016) no.5, 059901] doi:10.1103/PhysRevC.91.034907, 10.1103/PhysRevC.94.059901 [arXiv:1410.3914 [physics.data-an]].

[17] M. Kitazawa, Phys. Rev. C 93 (2016) no.4, 044911 doi:10.1103/PhysRevC.93.044911 [arXiv:1602.01234 [nucl-th]].

[18] A. Bzdak, R. Holzmann and V. Koch, Phys. Rev. C 94 (2016) no.6, 064907 doi:10.1103/PhysRevC.94.064907 [arXiv:1603.09057 [nucl-th]].

[19] J. Brewer, S. Mukherjee, K. Rajagopal and Y. Yin, Phys. Rev. C 98 (2018) no.6, 061901.

[20] P. Bożek, W. Broniowski, M. Rybczynski and G. Stefanek, arXiv:1901.04484 [nucl-th].

[21] M. Rybczynski, G. Stefanek, W. Broniowski and P. Bożek, Comput. Phys. Commun. 185 (2014) 1759.

[22] W. Broniowski, M. Rybczynski and P. Bożek, Comput. Phys. Commun. 180 (2009) 69.

[23] B.I. Abelev et al. [STAR collaboration], Phys. Rev. C 79 (2009) 034909

[24] L. Adamczyk et al. [STAR collaboration], Phys. Rev. C 96 (2017) 044904

[25] C. A. Pruneau, arXiv:1903.04591 [nucl-th].

[26] J. Brewer, talk at this conference.

[27] X. Luo, Nucl. Phys. A 956 (2016) 75. 